\title{
Operando Untersuchungen der gas-sensorischen Eigenschaften von Metallphthalocyaninen durch kombinierte elektrische, optische und gravimetrische Messungen
}

\author{
Wolfram Simmendinger, Alexandru Oprea, Nicolae Barsan, Udo Weimar \\ Institut für physikalische und theoretische Chemie, Universität Tübingen, Auf der Morgenstelle15, \\ 72076 Tübingen,
}

\section{Zusammenfassung}

Aus verschiedenen Metallphthalocyanine ( $\mathrm{CoPc}, \mathrm{ZnPc}, \mathrm{TiOPc})$ für gassensorische Anwendungen wurden durch Vakuumsublimation Sensoren hergestellt und, unter operando (betriebsähnliche) Bedingungen untersucht. Die Austrittsarbeit-, Impedanzspektroskopie-, Gleichstromleitfähigkeits-, FTIR-Transmissionsspektroskopie-, und gravimetrischen-Messungen haben eine hohe Empfindlichkeit gegenüber kleinen $\mathrm{NO}_{2}$-Konzentrationen dokumentiert. Die starke Wechselwirkung zwischen dem Analyten und den empfindlichen Schichten hat zu einer guten Gasempfindlichkeit geführt, aber auch zu relativ langen Desorptionszeiten. Deswegen eignen sich diese Materialien für Anwendungen die eine niedrige Nachweisgrenze für Einzelereignisse benötigen.

\section{Experimentelles, Ergebnisse und Diskussion}

Seit der Beobachtung, dass Phthalocyanine (Pc) ihre elektrischen Eigenschaften durch Ab- bzw. Desorption von Gasen ändern, wurden große Anstrengungen unternommen diese als sensitive Schichten für chemische Sensoren $\mathrm{zu}$ verwenden, wobei insbesondere für oxidierende Gase, wie zum Beispiel $\mathrm{NO}_{2}, \mathrm{Cl}_{2}$ und $\mathrm{O}_{3}$ gute Resultate erzielt werden konnten [1, 2, 3]. Neben der großen thermischen und chemischen Stabilität besteht die Möglichkeit, durch Veränderung des Zentralatoms, der Gitterstruktur bzw. der organischen Seitenketten die Selektivität und das Ansprechverhalten gegenüber dem Analyten zu erhöhen.

Zur Realisierung dessen sind kombinierte Operando-Messungen notwendig, um ein besseres Verständnis der Vorgänge auf mikroskopischer Ebene zu ermöglichen, da bis jetzt nur Untersuchungen durchgeführt wurden, in denen nur wenige dieser Methoden gleichzeitig zur Anwendung kamen. Zu diesem Zweck wurden verschiedene Metallphthalocyanine (MPc) im selben Arbeitsschritt im Hochvakuum auf die jeweiligen Substrate aufgedampft, um für die verschiedenen Messungen identische Schichten zu erhalten. Diese wurden mittels Leitfähigkeits-, Kontaktpotential-, Impedanzspektroskopie-, Infrarot-, UV/VIS- und Gravimetriemessungen untersucht, wobei sie verschiedenen Konzentrationen von $\mathrm{NO}_{2}$ und Luftfeuchtigkeit ausgesetzt wurden. Die Ergebnisse dieser Messungen werden analysiert und sollen dazu beitragen bestehende Modelle zu verfeinern und ein besseres Verständnis der Mechanismen, die zu einer hohen Gasempfindlichkeit führen, zu erreichen

In Bild 1 und 2 sind die Änderungen Kontaktpotentialdifferenzen von $\alpha$-CoPc, $\alpha$-ZnPc sowie amorphem und $\alpha$ TiOPc bei Exposition zu $\mathrm{NO}_{2}$ zu sehen. Durch das oxidierende $\mathrm{NO}_{2}$ werden negative Änderungen verursacht, während unter einem Feuchtigkeitshintergrund, welcher den reduzierenden Charakter von $\mathrm{H}_{2} \mathrm{O}$ besitzt, positive Änderungen durch $\mathrm{NO}_{2}$ beobachtet werden können. Die untersuchten MPcs zeigen hier ein ähnliches Verhalten, wobei die Effekte bei dem auf $50^{\circ} \mathrm{C}$ geheizten $\alpha$-ZnPc stärker ausgeprägt sind, als bei $\alpha$-CoPc und $\alpha$-TiOPc. Bei den Messungen der Leitfähigkeit (Bild 3) fällt die sehr hohe Empfindlichkeit von amorphem TiOPc auf, wobei diese Schicht unter Einfluss von Feuchtigkeit deutlich geringere Empfindlichkeit zeigt und sich zudem als nicht langzeitstabil erwies, was im Fall von geordnetem $\alpha$-TiOPc gegeben war.

Es ist bereits bekannt, dass z.B. CuPc und $\mathrm{PbPc}$ Eigenschaften aufweisen, die sie geeignet zur Detektion von $\mathrm{NO}_{2}$ machen, allerdings sind die Ansprech- und Relaxationszeiten sowie die Langzeitstabilität oft unbefriedigend. Nichtsdestotrotz zeigen auch weniger untersuchte Metallphthalocyanine gute Voraussetzungen 
zur $\mathrm{NO}_{2}$-Detektion; neben einer hohen Empfindlichkeit und verhältnismäßig kurzen Ansprechzeit, bereits bei moderaten Temperaturen, verliefen auch erste Tests der Langzeitstabilität vielversprechend, so dass, beispielsweise TiOPc oder $\mathrm{ZnPc}$, je nach Sensorprinzip, für konkrete Anwendungen, wie z.B. Alarmierung bei Grenzwertüberschreitung, geeignet erscheinen.

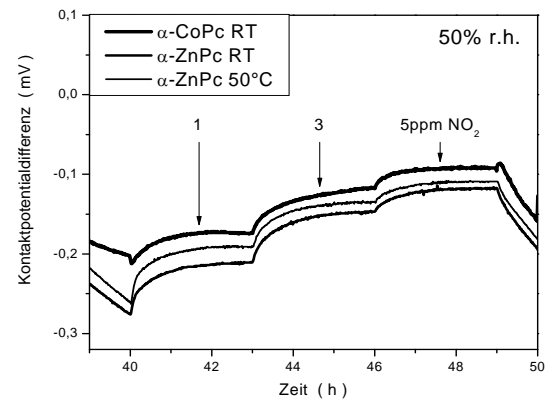

Bild 1 Kontaktpotentialdifferenz von $\mathrm{CoPc}$ und $\mathrm{ZnPc}$ unter $\mathrm{NO}_{2}$-Einfluss

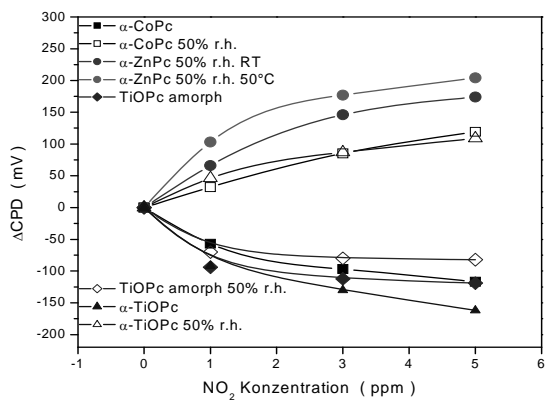

Bild 2 Änderung der Kontaktpotentialdifferenzen von $\mathrm{CoPc}, \mathrm{ZnPc}$, und TiOPc unter Exposition zu $\mathrm{NO}_{2}$

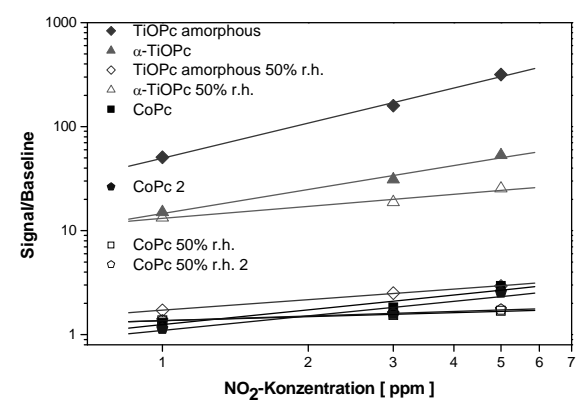

Bild 3 Signale der Leitfähigkeitsmessung von CoPc und TiOPc bei Exposition zu $\mathrm{NO}_{2}$.

\section{Literatur}

[1] M. Fleischer, Detection of volatile compounds correlated to human diseases through breath analysis with chemical sensors, Sensors and Actuators B (2002) S. 245-249.

[2] T. Miyata, High sensitivity chlorine gas sensors using $\mathrm{Cu}$-phthalocyanine thin films, Thin Solid Films, 425 (2003) S. 255-259.

[3] A. Oprea,, Copper phthalocyanine suspended gate field effect transistors for NO2 detection, Sensors and Actuators B, 118 (2006) S. 249-254. 